\title{
GCU
}

Glasgow Caledonian

University

University for the Common Good

\section{Partial discharge pulse propagation in power cable and partial discharge monitoring system}

Sheng, Bojie; Zhou, Chengke; Hepburn, Donald; Dong, Xiang ; Peers, Graham; Wenjun, Zhou; Tang, Zeyang

Published in:

IEEE Transactions on Dielectrics and Electrical Insulation

DOI:

10.1109/TDEI.2014.6832236

Publication date:

2014

Document Version

Author accepted manuscript

Link to publication in ResearchOnline

Citation for published version (Harvard):

Sheng, B, Zhou, C, Hepburn, D, Dong, X, Peers, G, Wenjun, Z \& Tang, Z 2014, 'Partial discharge pulse propagation in power cable and partial discharge monitoring system', IEEE Transactions on Dielectrics and Electrical Insulation, vol. 21, no. 3, pp. 948-956. https://doi.org/10.1109/TDEl.2014.6832236

\section{General rights}

Copyright and moral rights for the publications made accessible in the public portal are retained by the authors and/or other copyright owners and it is a condition of accessing publications that users recognise and abide by the legal requirements associated with these rights.

Take down policy

If you believe that this document breaches copyright please view our takedown policy at https://edshare.gcu.ac.uk/id/eprint/5179 for details of how to contact us. 


\title{
Partial Discharge Pulse Propagation in Power Cable and Partial Discharge Monitoring System
}

\author{
Bojie Sheng, Chengke Zhou, Donald M Hepburn, Xiang Dong \\ Glasgow Caledonian University \\ School of Engineering and Built Environment \\ Glasgow, G4 0BA, UK \\ Graham Peers \\ EDF Energy \\ East Killbride, G74 5PG, UK \\ Wenjun Zhou and Zeyang Tang \\ Wuhan University \\ School of Electrical Engineering \\ Wuhan, 430072, China
}

\begin{abstract}
Partial discharge (PD) based condition monitoring has been widely applied to power cables. However, difficulties in interpretation of measurement results (location and criticality) remain to be tackled. This paper aims to develop further knowledge in PD signal propagation in power cables and attenuation by the PD monitoring system devices to address the localization and criticality issues. As on-line or in-service PD monitoring sensors commonly comprise of a high frequency current transformer (HFCT) and a high-pass filter, the characteristics of detected PD pulses depend on the attenuation of the cable, the HFCT used and the filter applied. Simulation of pulse propagation in a cable and PD monitoring system are performed, based on analyses in the frequency domain using the concept of transfer functions. Results have been verified by laboratory experiments and using on-site PD measurements. The knowledge gained from the research on the change in pulse characteristics propagating in a cable and through a PD detection system can be very useful to PD denoising and for development of a PD localization technique.
\end{abstract}

Index Terms - Cable, Partial discharge, on-line monitoring, transfer function, denoising, localization.

\section{INTRODUCTION}

POWER cables are subject to electrical, thermal, mechanical, and environmental stresses on a constant basis when in service. These stresses, and occasionally problems resulting from inadequate installation and maintenance practices, lead to insulation degradation or defects $[1,2]$. In many instances the deterioration leads to partial discharge (PD) which exacerbates the degradation of the insulation and shortens the cable life [3, 4]. Although it has been reported that, in cable systems, most of the PDs occur at joints and terminations [5], locating failures is still a challenge. Detection of incipient faults through PD monitoring is therefore of great importance as it allows timely maintenance and replacement of assets to be carried out and unplanned outage to be reduced [6]. Consequently, on-line PD monitoring has been widely applied to power cables to reveal these defects and to assess their criticality $[7,8]$.
On-line PD monitoring is often carried out through high frequency current transformers (HFCT) being installed at cable joints or terminations where the HFCTs can be clipped around the cable earth bond leads [9] and high-pass filters (HPFs) applied to reduce the effects of noise [10]. Although technologies in data acquisition and signal processing have seen good advancement over the years [8], challenges remain in localizing the source of PD activity.

Although many publications have reported utilizing a HFCT and a HPF in on-line PD detection, e.g. $[8,11]$, no report has so far discussed the difference between the original pulse and the recorded pulse resulting from both cable attenuation and the effects of the PD detection instrument. A good understanding and utilization of induced charge is crucial for effective PD denoising and PD recognition when both HFCT and HPF are employed. Great efforts have been made in understanding the attenuation of pulses during propagation in cables, to determine the change in 
the characteristic amplitude of a pulse travelling in cables [12, 13]. However, there is a fundamental problem about the characteristic of the shape of the pulse as it propagates in cables which has not been addressed, where this knowledge is useful for PD recognition and localization, for a given fault location in a cable it is understood that although the original pulse amplitude is changeable the shape of the pulse is consistent [14].

In the present paper, further knowledge is developed to understand PD signal propagation in cables and the effects of the PD monitoring system. The on-site monitoring system considered includes the use of sensors, each comprised of a HFCT and a HPF. The characteristics of the detected PD pulse, as compared with the pulse at the PD site of origin depends on the attenuation properties of the cable, HFCT and HPF. Simulation of pulse propagation in cable and PD detection components, based on analysis in the frequency domain using transfer functions of cables, HFCT and HPF are performed, and results are verified by laboratory experiments.

\section{PD PULSE PROPAGATION IN CABLES}

\subsection{THEORETICAL BACKGROUND}

The power cable, behaving as a transmission line to a travelling wave or signal can be described by an equivalent circuit with distributed parameters per unit length, resistance $R(\Omega / \mathrm{m})$, inductance $L(\mathrm{H} / \mathrm{m})$, conductance $G(\mathrm{~S} / \mathrm{m})$, and capacitance $C$ $(\mathrm{F} / \mathrm{m})$, which is shown in Figure 1 [15].

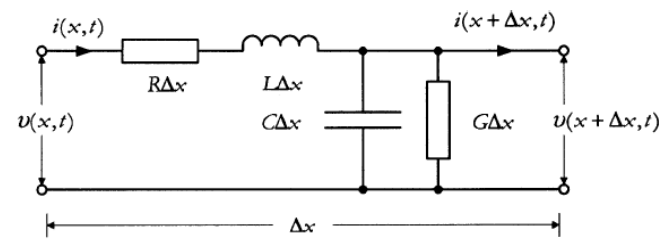

Figure 1. Equivalent circuit representation of a transmission line element of length $\Delta \mathrm{x}$

Inductive and capacitive circuit parameters are frequency dependent, where frequency is given by $f(\mathrm{~Hz})$. The line is described by its propagation constant $\gamma$ with attenuation constant $\alpha(\mathrm{dB} / \mathrm{m})$ as real and phase constant $\beta(\mathrm{rad} / \mathrm{m})$ as the imaginary part. The following relation holds:

$$
\gamma(\omega)=\alpha(\omega)+j \beta(\omega)=\sqrt{(R+j \omega L)(G+j \omega C)}
$$

Where: $\omega=2 \pi f$ is angular frequency ( $\mathrm{rad} / \mathrm{sec})$

As different frequencies of a pulse travel along a cable, they suffer different degrees of attenuation and dispersion. Attenuation $\alpha$ is due to losses in the solid dielectric and propagation through the resistance of the conductor, sheath and semi-conducting layers. Normally, attenuation severity increases with frequency. Dispersion is a phenomenon whereby different frequency signals travel along a cable at different speeds, which results in frequency-dependent phase shifts. Therefore, it can be said that attenuation and dispersion will decrease the amplitude and increase the width of the pulse, hence changing the rise time, as the pulse propagates along a cable [16].

\subsection{CABLE TRANSFER FUNCTION}

The propagation constant of a wave travelling along a transmission line of segment length $l$ is the complex ratio between the voltages of output (transmitted) pulse $V_{\text {out }}$ and the input (incident) pulse $V_{\text {in }}$ of the line segment. If a length of cable is considered as a linear system, this ratio represents the cable Transfer Function $H(\omega)$ as:

$H(\omega)=\frac{V_{\text {out }}}{V_{\text {in }}}=e^{-r(\omega) 2 l}=e^{-(\alpha(\omega)+j \beta(\omega)) 2 l}$

Although the input pulse and output pulse cannot be measured directly due to existence of mismatched impedance between the samples and instrumentation, a calibration procedure has been developed to obtain $H(\omega)$ [17]. Set-up of the procedure can be discussed using Figure 2. As in [17], in the following equations the forward and backward travelling waves are indexed with "+" and "-" respectively, and the index numbers in Figure 2 specify the points where the voltages are calculated. Initially line 2 is short-circuited and the measured signal from this configuration is $V_{-3}{ }^{\text {short }}$, thereafter the device under test (DUT) is connected to line 2 and the data collected. Using Fast Fourier Transform (FFT), the pulses are then transferred to the frequency domain, where the transfer function of the system can be obtained from [17]:

$H(\omega)=e^{-r(\omega) 2 l}=\frac{V_{-3} V_{-3}^{\text {short }}}{\left(V_{-3}^{m}\right)^{2}-\left(V_{-3}^{\text {short }}\right)^{2}}$

Where $V_{-3}$ is the DUT response, $V_{-3}{ }^{\text {short }}$ is the measured signal reflected at the short-circuited end of coaxial line 2 and $V_{-3}{ }^{m}$ is the mismatch reflection.

Pulse injection and

measurement system

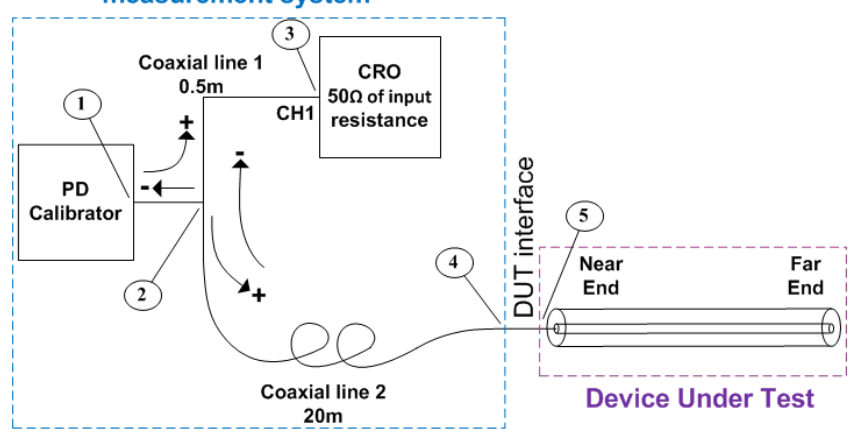

Figure 2. Schematic drawing of a calibration procedure setup

The attenuation and phase constant can be deduced from (3) as

$\alpha(\omega)=-\frac{1}{2 l} \ln |H(\omega)|$
$\beta(\omega)=-\frac{1}{2 l} \angle H(\omega)$

Following the calibration procedure, an experiment was carried out by injecting a calibration pulse shown in Figure 3 into a $38.8 \mathrm{~m} 11 \mathrm{kV}$ single core Ethylene-Propylene-Rubber (EPR) 
insulated cable specimen, which consists of copper conductor, EPR polymeric insulation, copper tape concentric screen and PVC sheath, to obtain the transfer function of the EPR cable. The measured attenuation and phase constant are given in Figure 4.

Results shown in Figure 4 indicate that the cable behaves like a transmission line up to $80 \mathrm{MHz}$, beyond this the characteristics becomes complicated and the cable cannot be represented using the transfer function due to the limitations in the frequency content of the input signal and the capabilities of the measurement equipment.

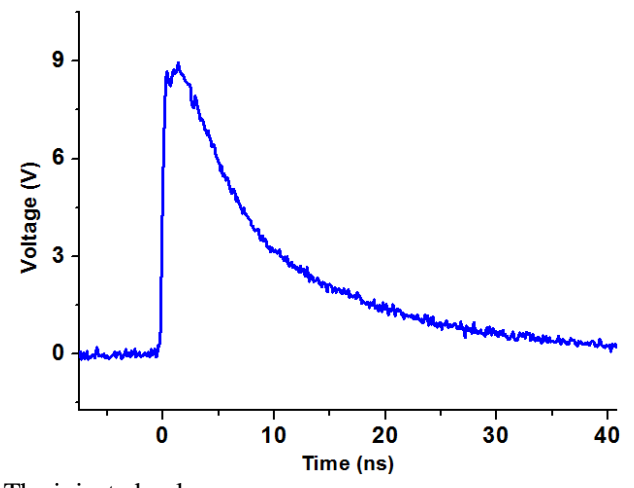

Figure 3. The injected pulse

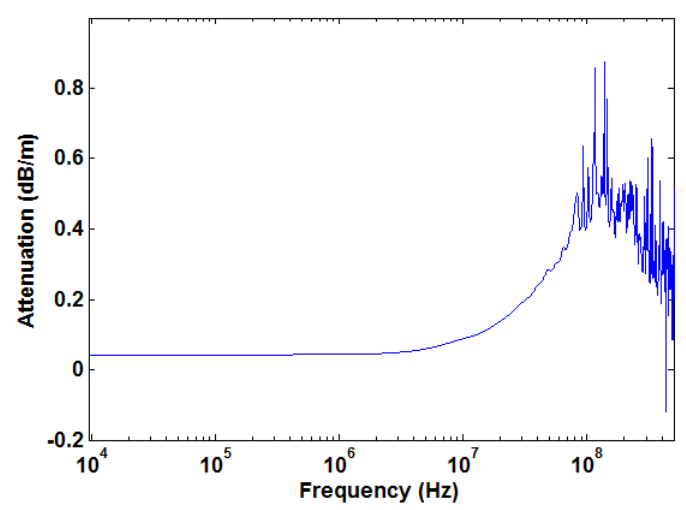

(a) Attenuation constant

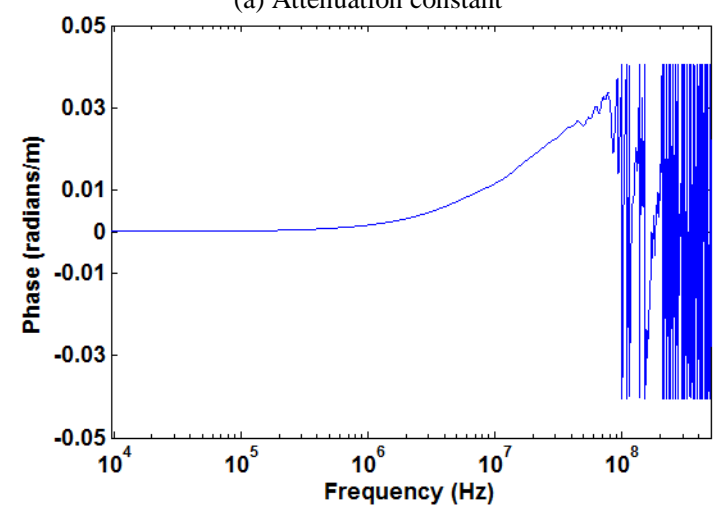

(b) Phase constant

Figure 4. Measured results of attenuation and phase constant of an $11 \mathrm{kV}$ EPR insulated cable

\subsection{SIMULATION OF PULSE PROPAGATION IN CABLES}

According to [16], a PD pulse can be approximated as a double exponential function defined by equation (6):

$V(t)=V_{0}\left(-\exp \left(-t / \tau_{1}\right)+\exp \left(-t / \tau_{2}\right)\right)$
Where: $\tau_{1}, \tau_{2}=$ time constants (s)

$$
V_{0}=\text { amplitude constant }(\mathrm{V})
$$

The shape of a pulse can be characterised by rise time $T_{r}$ (rise from $10 \%$ to $90 \%$ of the peak) and pulse width $T_{F W H M}$ (full width at half maximum), as shown in Figure 5.

Simulations of pulse propagation in an $11 \mathrm{kV}$ EPR cable have been made. The methodology for carrying out the simulation can be summarized briefly as follows: Firstly, a double exponential pulse is assumed as an incident pulse $V_{i n}(t)$ which has been generated at one end of the cable with a length of L. Secondly, the incident pulse is converted into the frequency domain $S_{i n}(\omega)$ via FFT. Thirdly, the output pulse from the other end of the cable in frequency domain $S_{\text {out }}(\omega)$ is determined by multiplying the cable transfer function $H(\omega)$ and $S_{i n}(\omega)$, Finally, the output pulse in time domain $V_{\text {out }}(t)$ can be obtained by Inverse FFT. Lastly, the shape parameters of the output pulse: $T_{r}$ and $T_{F W H M}$ can be extracted. As the frequency spectrum of a pulse dictates the pulse shape, the shape of a detected pulse for a given cable is only affected by the shape of the incident pulse and the frequency dependent attenuation during propagation. To overcome the impact of magnitude changes which occur during propagation, but which are not pertinent to the frequency spectrum, all pulse amplitudes are normalised to $1 \mathrm{~V}$ in the following simulations.

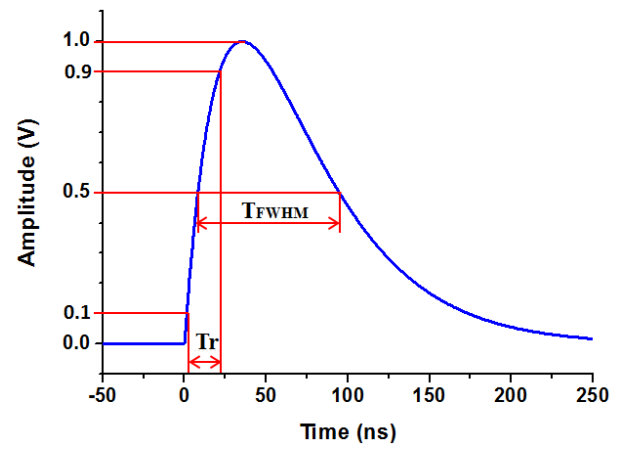

Figure 5. Illustration of $T r$ and $T_{F W H M}$ of a PD pulse

\subsubsection{Pulse propagation in cables with different length}

A simulation for $11 \mathrm{kV}$ EPR cable with its length varying from $0 \mathrm{~m}$ to $200 \mathrm{~m}$ was carried out. Based on the known properties of the injected pulse and calibration measurements the input pulse, or incident pulse, is taken to have $1.3 \mathrm{~ns}$ rise time and $9.5 \mathrm{~ns}$ $T_{\text {FWHM }}$. Simulation results, Figure 6 , demonstrate that the output pulse rise time and pulse width increase monotonically with length of cable over which the pulse has propagated.

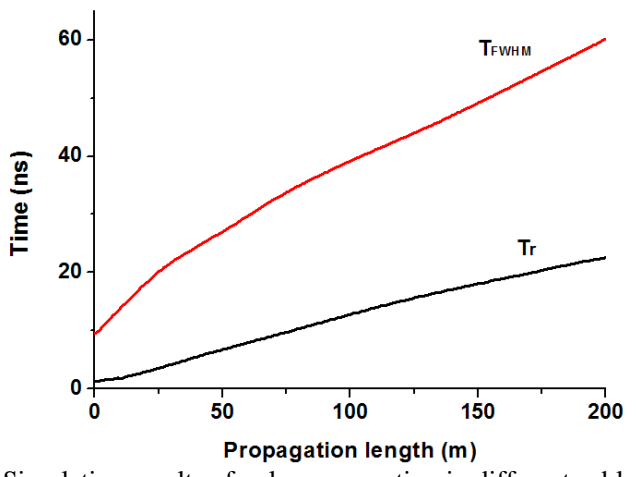

Figure 6. Simulation results of pulse propagation in different cable lengths 


\subsubsection{Bandwidth of pulse propagation along a cable}

As a PD pulse propagates along a cable its high frequency components suffers greater attenuation, resulting in a reduction in the optimum bandwidth for detection which can be taken as roughly the $-6 \mathrm{~dB}$ bandwidth of the detection device [18]. Another simulation of a pulse with $T_{r}$ of $1 \mathrm{~ns}$ and $T_{F W H M}$ of $5 \mathrm{~ns}$ being assumed to travel along a cable was performed. In fact, the rise time of observed original PD pulse emanating from any insulation defect is about $1 \mathrm{~ns}[19,20,21]$. The result of the relationship between the upper limit of the bandwidth of the pulse with the propagation length is shown in Figure 7. It depicts that pulse bandwidth becomes limited to $20 \mathrm{MHz}$ after a pulse travels approximately $50 \mathrm{~m}$. This indicates that the detected pulse shape would be modified by the sensors if the source of PD is shorter than $50 \mathrm{~m}$ away from the sensor. This results from limitations of the sampling resolution of online monitoring system and frequency response of the HFCT. As the sampling resolution of the PD monitoring system used is $100 \mathrm{MS} / \mathrm{s}$ it has a resolution of 10ns/point, therefore the PD monitoring system impacts on for the ability to detect a pulse rise time. This limitation corresponds to the shape of a pulse which has travelled approx. 50 metres. Although the limitation of the sampling resolution of instrument can be overcome by improvement of hardware used in industrial applications, the limitation of frequency response of HFCT cannot be improved easily. As will be discussed later in the paper, HFCTs which possess a bandwidth range of $50 \mathrm{kHz}-20 \mathrm{MHz}$ are commonly adopted for PD detection.

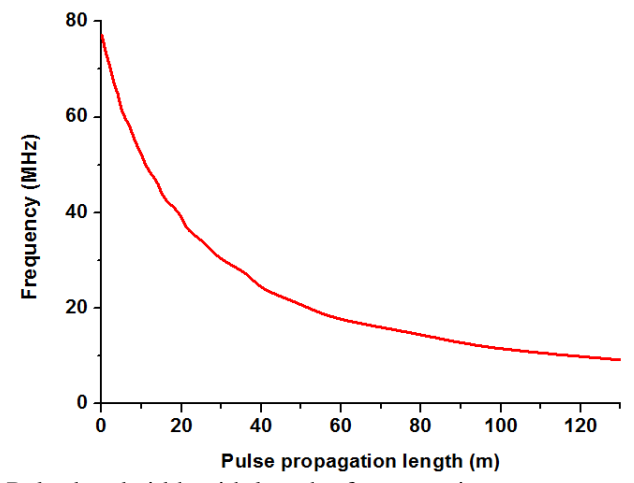

Figure 7. Pulse bandwidth with length of propagation

\subsubsection{The shape of pulse propagation along a cable}

In order to investigate the shape of a pulse propagating along a cable, pulses with the same $T_{r}(1 \mathrm{~ns})$ and different $T_{F W H M}$, as shown in Table 1, were assumed in a series of simulation studies. It can be observed from the results shown in Table 2 that $T_{r}$ and $T_{F W H M}$ of output pulses increase monotonically while the original pulses have the same $T_{r}$ and different $T_{F W H M}$. As $T_{r}$ is consistent in all pulses their high frequency content will be very similar, hence the high frequencies will be attenuated consistently. A larger pulse width, $T_{F W H M}$, has a narrower frequency bandwidth, as the attenuation constant of the cable is consistent at lower frequencies the width of the output pulse is changing as expected.

Table 1. Parameters of different original pulses

\begin{tabular}{c|c|c}
\hline & $T_{r}(\mathrm{~ns})$ & $T_{F W H M}(\mathrm{~ns})$ \\
\hline \multirow{2}{*}{ Original pulses } & 1 & 3 \\
& & 4 \\
\hline
\end{tabular}

Table 2. Simulation results of pulses with the same $T_{r}$ and different $T_{F W H M}$ (a) Rise time

\begin{tabular}{ll|lllll}
\hline \multicolumn{5}{c|}{ Original pulse } & \multicolumn{5}{c}{$T_{r}(\mathrm{~ns})$ of output pulse from different length cables } \\
$\begin{array}{l}T_{r} \\
(\mathrm{~ns})\end{array}$ & $\begin{array}{l}T_{F W H M} \\
(\mathrm{~ns})\end{array}$ & $100 \mathrm{~m}$ & $200 \mathrm{~m}$ & $300 \mathrm{~m}$ & $500 \mathrm{~m}$ & $1000 \mathrm{~m}$ \\
\hline \multirow{3}{*}{1} & 3 & 8.1 & 17.5 & 25.5 & 45.4 & 104.5 \\
& 4 & 8.8 & 17.8 & 25.7 & 45.5 & 104.6 \\
& 5 & 9.5 & 18.3 & 26.1 & 45.8 & 104.7 \\
\hline
\end{tabular}

(b) Pulse width

\begin{tabular}{ll|lllll}
\hline \multicolumn{5}{c|}{ Original pulse } & \multicolumn{5}{c}{$T_{F W H M}(\mathrm{~ns})$ of output pulse from different length cables } \\
$\begin{array}{l}T_{r} \\
(\mathrm{~ns})\end{array}$ & $\begin{array}{l}T_{F W H M} \\
(\mathrm{~ns})\end{array}$ & $100 \mathrm{~m}$ & $200 \mathrm{~m}$ & $300 \mathrm{~m}$ & $500 \mathrm{~m}$ & $1000 \mathrm{~m}$ \\
\hline \multirow{3}{*}{1} & 3 & 24.7 & 47 & 75.2 & 135.5 & 227.4 \\
& 4 & 25.3 & 47.3 & 75.4 & 135.9 & 227.4 \\
& 5 & 26.5 & 48 & 75.8 & 136.5 & 227.5 \\
\hline
\end{tabular}

\subsection{EXPERIMENTAL VERIFICATION}

Laboratory experiments were carried out on a $38.8 \mathrm{~m} 11 \mathrm{kV}$ EPR cable to verify the simulation results. An oscilloscope with a sampling rate of $10 \mathrm{GS} / \mathrm{s}$, a vertical resolution of 8 bits, and a bandwidth of $3 \mathrm{GHz}$ has been used for data acquisition. A PD calibrator which can generate a pulse with 1 ns rise time was used in the experiment. As it is difficult to capture the input pulse and output pulse simultaneously, due to impedance mismatch between the cable and the measurement system, the experiments had to be specifically designed to ignore the effect of the pulse reflection as a pulse entered the cable sample. Experimental setups are shown in Figure 8. In both the set-ups, the PD calibrator was connected to two lengths of coaxial cable by a T-connection. In set-up $1,0.5 \mathrm{~m}$ long coaxial cable 1 is connected to the PD calibrator while the other end connects to channel 1 of the oscilloscope by a $10 \mathrm{~dB}$ attenuator, $20 \mathrm{~m}$ long coaxial cable 2 is connected to channel 2 using a $10 \mathrm{~dB}$ attenuator. In set-up two, cable 1 connects between the PD calibrator and the oscilloscope as previously, cable 2 connects to one end of the EPR cable under test. The other end of the cable connects to channel 2 of the scope using $0.5 \mathrm{~m}$ long coaxial cable 3 .

The experimental results are depicted in Figure 9, where:

$V_{11}$ - pulse detected in channel 2 in set-up one.

$V_{21}$ - first pulse detected in channel 2 in set-up two.

$V_{23}$ - third pulse detected in channel 2 in set-up two.

$V_{24}$ - fourth pulse detected in channel 2 in set-up two.

In Figure 9a the first pulse is that detected at channel 1. $V_{l l}$ is the pulse detected at channel 2 and, thus, the pulse injected into the end of the DUT in the second set-up.

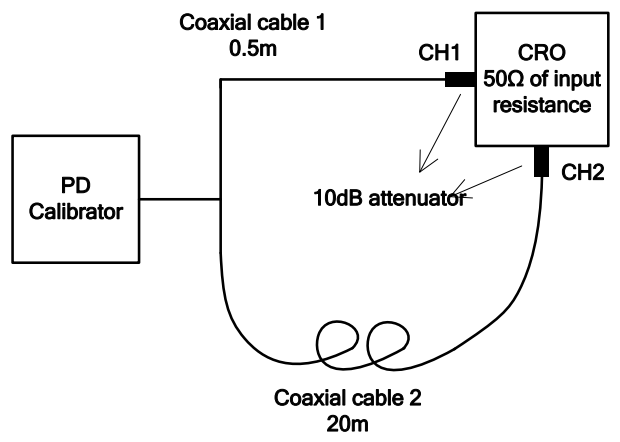

(a) Set-up one 
Pulse injection and measurement system

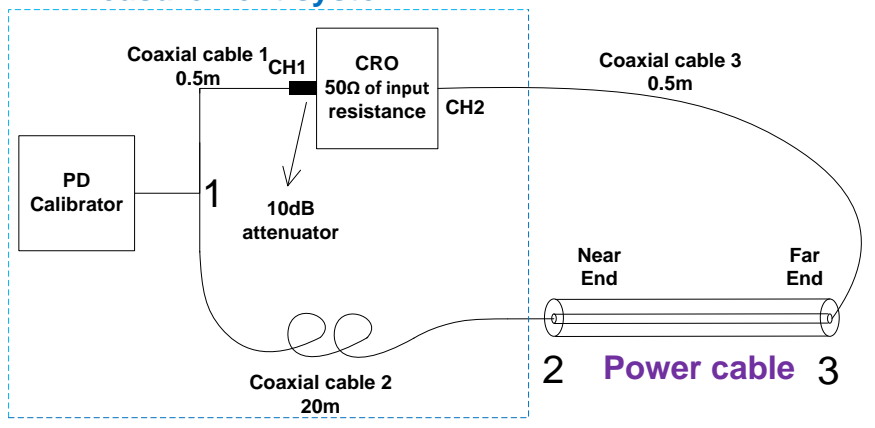

(b) Set-up two

Figure 8. Experiment set-ups in laboratory

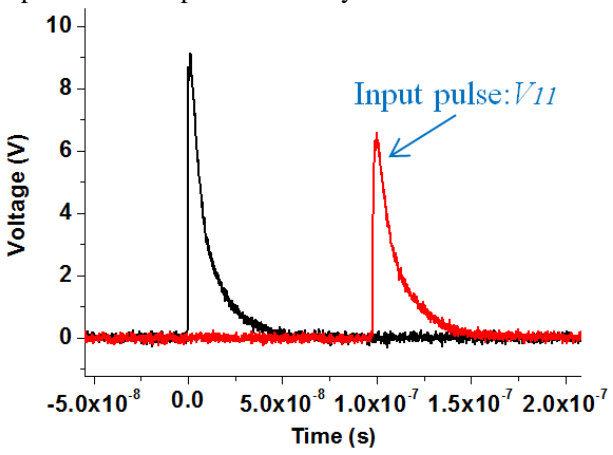

(a) Result of set-up one

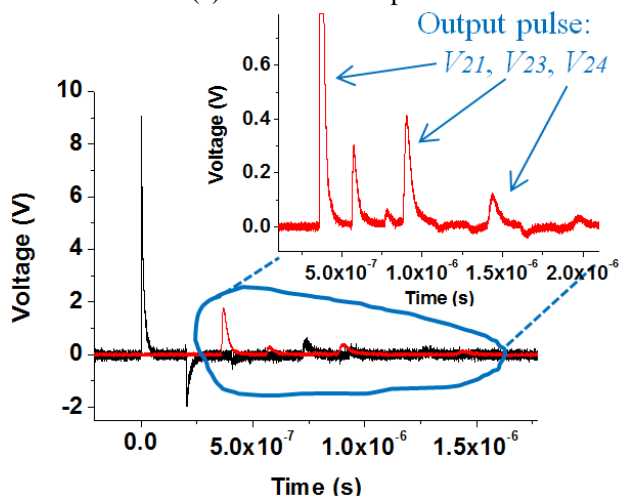

(b) Result of set-up two

Figure 9. Experimental results

The shape of the incident pulse injected into the cable in set-up two can be considered the same as that of $V_{l l}$. At the start of the cable, due to impedance mismatch, part of the signal will be reflected but the pulse shape will be maintained as the reflection coefficient is independent of frequency. The output pulse detected at channel 2 will be proportional to the signal at the cable end but the magnitude will be reduced. The shape, not the magnitude, of $V_{21}, V_{23}$ and $V_{24}$ are considered in the output pulses from the cable, attenuation of coaxial cable 3 is ignored. The propagation lengths of $V_{21}, V_{23}$ and $V_{24}$ are, respectively, one, three and five times the length of the cable under test. $V_{2 l}$ magnitude is clipped in the expanded region to give clear identification of lower magnitude peaks.

Simulations have been carried out for the pulse $V_{11}$ to travel one times, three times and five times of the length of the EPR cable individually. A comparison of normalized output pulses resulting from simulation and those from experimental results are given in Figure 10. It can be observed that simulation results are in good agreement with experimental output pulses for all three cable lengths, i.e. $38.8 \mathrm{~m}, 116.4 \mathrm{~m}$ and $194 \mathrm{~m}$. Due to limit of cable length in laboratory, simulation beyond the range cannot be verified.

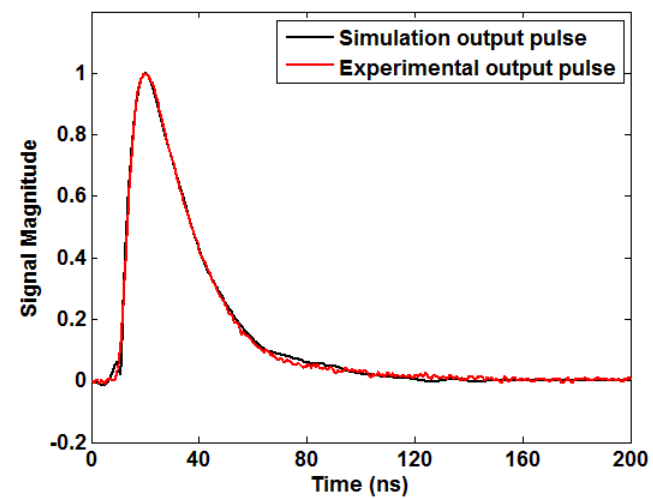

(a) Comparison of simulated and practical pulse - propagated $38.8 \mathrm{~m}$

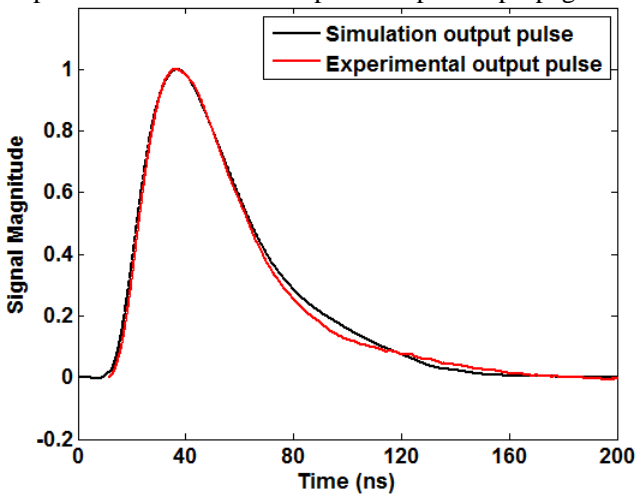

(b) Comparison of simulated and practical pulse - propagated $116.4 \mathrm{~m}$

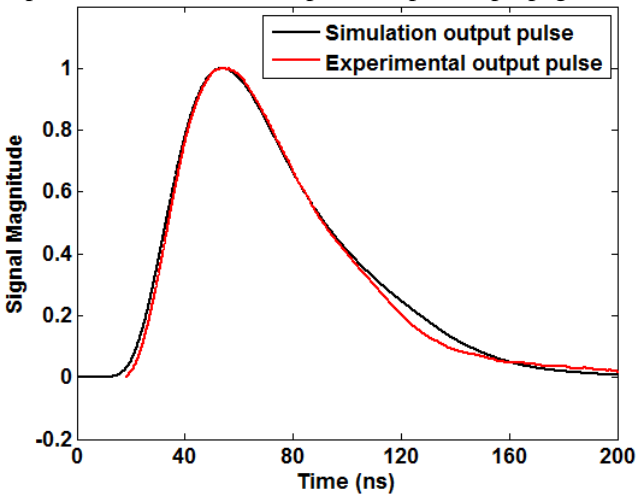

(c) Comparison of simulated and practical pulse - propagated $194 \mathrm{~m}$ Figure 10. Comparison between simulation and experimental results for pulse propagation in different cable lengths

\section{PULSE ATTENUATION IN HFCT AND FILTER}

\subsection{FREQUENCY RESPONSE OF HFCT}

A high frequency current transformer (HFCT) is designed specifically for picking up partial discharge current signals. It has a split ferrite core to allow retrospective fitting to earth bond lead without the need for disconnection of the cable under test. A typical HFCT for on-site PD detection has a frequency range of $50 \mathrm{kHz}$ to $20 \mathrm{MHz}$ with the transfer function of the HFCT $100 / 50$ $\left(H_{H F C T}(\omega)\right)$, shown in Figure 11. The transfer function was developed from equation (7) experimentally. The experiments used the HFCT to detect a high frequency pulse and a low 
frequency square signal separately. Then two transfer functions were obtained by equation (7). As the first function has high accuracy in high frequency section and low accuracy in low frequency section resulting from the high frequency input pulse, while the second function has low accuracy in high frequency section and high accuracy in low frequency section because of the low frequency input signal, the results of the first function in high frequency section and the results of the second function in low frequency section were combined to obtain the accurate transfer function of the HFCT, which is in good agreement with that in the data sheet of HFCT 100/50. Frequency and phase responses are shown in Figures $11 \mathrm{a}$ and $11 \mathrm{~b}$ respectively.

$$
H_{H F C T}(\omega)=\frac{V_{\text {out }}}{I_{\text {in }}}
$$

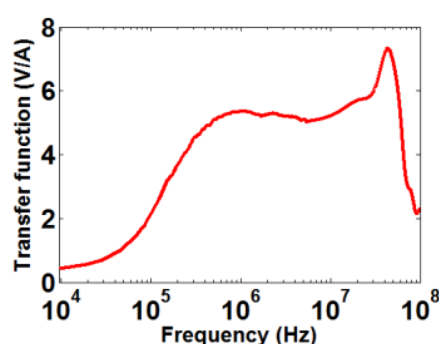

(a) Frequency response

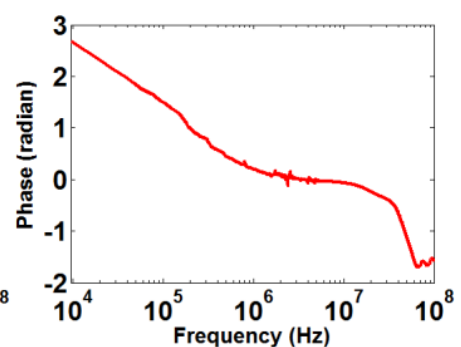

(b) Phase response
Figure 11. Experimental data of transfer function of HFCT100/50

\subsection{FREQUENCY RESPONSE OF FILTER}

A first-order resistance-capacitance (RC) high-pass filter (HPF) is usually used as a PD filter in on-line monitoring system as PD signals contain high frequencies they are able to pass through the filter, while mains frequencies and lower harmonics are blocked. The schematic circuit diagram of high-pass filter is given in Figure 12 and the value of resistance $R$ and capacitance $C$ are $50 \Omega$ and $0.01 \mu \mathrm{F}$ respectively, giving a cut-off frequency of $318 \mathrm{kHz}$. The transfer function of the HPF is given in equation (8) below:

$$
H_{H P F}(\omega)=\frac{V_{\text {out }}}{V_{\text {in }}}=\frac{1}{1-j(1 / \omega R C)}
$$

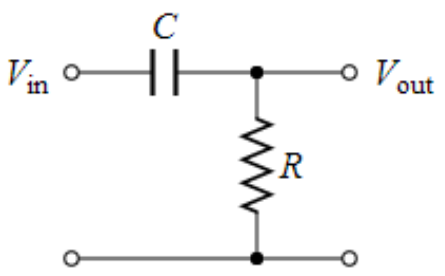

Figure 12. Schematic circuit diagram of high-pass filter

\subsection{ATTENUATION IN HFCT AND THE FILTER}

Simulation of the response of the HFCT and HPF to a known current pulse was performed. In the simulation process, the original pulse is converted into the frequency domain $S_{\text {ori }}(\omega)$ via FFT with sampling rate of $10 \mathrm{GS} / \mathrm{s}, 21000$ data points and a rectangular window function. Then, the detected pulse by HFCT and HPF is determined in the frequency domain $S_{\text {det }}(\omega)$ by multiplying the system transfer function $H_{s y s}(\omega)$, given in
Equation (9), and $S_{\text {ori }}(\omega)$. From this the detected pulse in the time domain $V_{d e t}(t)$ can be obtained by Inverse FFT (IFFT).

$$
H_{\text {sys }}(\omega)=H_{H F C T}(\omega) * H_{H P F}(\omega)
$$

The original input pulse and the detected output pulse by simulation are shown in Figure 13. It can be observed that the output pulse, after passing through the HFCT and filter, is shown to be markedly different from the input pulse as there is an inverse peak after the initial positive peak in the output wave.

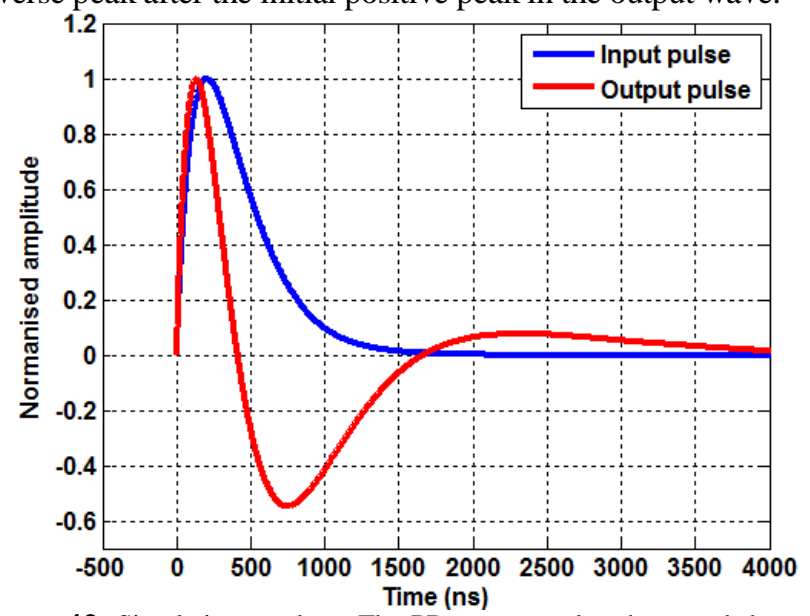

Figure 13. Simulation results - The PD current pulse shape and the pulse shape after passing through the PD detection system

\section{PULSE PROPAGATION IN CABLE AND PD MONITORING SYSTEM}

\subsection{CHARACTERISTICS OF DETECTED PULSES}

To investigate the characteristics of detected pulse attenuated by both the cable and the PD monitoring system, simulations combining pulse propagation with different cable lengths and PD monitoring system response were made. In the simulation procedure, a pulse with $T_{r}$ of $1 \mathrm{~ns}$ and $T_{F W H M}$ of $5 \mathrm{~ns}$ is assumed to have originated at one end of an $11 \mathrm{kV}$ EPR cable, then propagated along the cable until it is detected by the PD monitoring system (HFCT and HPF) at the other end of the cable. The results of simulation for cable lengths of $50 \mathrm{~m}, 100 \mathrm{~m}, 150 \mathrm{~m}$ and $200 \mathrm{~m}$ are depicted in Figure 14.

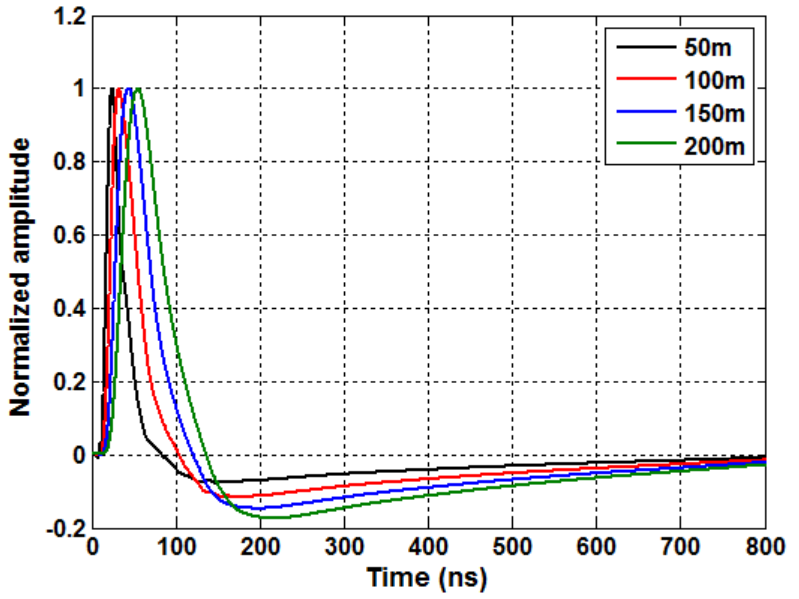

Figure 14. Simulation results of pulse propagation over different cable lengths detected by the PD monitoring system 
From Figure 14, the cable length is shown to affect the ratio of inverse peak to first peak of detected pulse. What's more, the further a pulse propagates, the greater is the ratio of the first inverse peak to the first peak of detected pulse (FIP-FP). This characteristic can potentially be developed for PD localization.

\subsection{ON-SITE MEASUREMENT OF PD PULSES}

To consider the validity of the simulation in the previous section, studies of actual cable data from on-site measurements have been made. A typical PD pulse detected on-site on an $11 \mathrm{kV}$ PILC underground distribution cable with an approximate length of $1000 \mathrm{~m}$ is given in Figure 15a, the measurements were taken with the cable in service. Figure $15 \mathrm{~b}$ shows a typical PD pulse from an on-line measurement on an $11 \mathrm{kV} 491 \mathrm{~m}$ EPR cable consisting of copper conductor, EPR polymeric insulation, copper tape concentric screen and PVC sheath in a power station in the UK. During the measurement the cable armour at the far end of the cable was insulated from earth, and solidly bonded to earth at the measurement end. From Figure 15 it can be observed that the predicted inverse peak is indicated after the first peak in the detected pulse. The magnitude of the inverse pulse is a little larger than the simulation but, given the noise inherent in the measurement is in good agreement with simulation results in Section 4.1.

The three pulse waveforms in Figure $15 \mathrm{~b}$ are believed to be from the same source, however, the propagation time will be dependent on the number of reflections before detection occurs. The first pulse will have propagated from the PD source within the $491 \mathrm{~m}$ cable to the PD detection system, the second pulse will have travelled an additional two times the cable length before detection, the third pulse will have travelled a further additional four times of the cable length. It can be observed that the time intervals between the pulses are each $6.5 \mu \mathrm{s}$. Thus, the pulse propagation velocity in the cable is $151 \mathrm{~m} / \mu \mathrm{s}$. During the PD measurement, the cable sheath at measurement end (near end) was solidly earthed and the far end of the cable was open circuited. Therefore, the characteristic impedance of the near end is smaller than that of the cable while that of the far end is considered infinite. According to the reflection law and the polarity of the three pulses, the PD was diagnosed as emanating from the far end of the cable.

Three sets of the measured pulses from the same cable, all being similar to that shown in Figure $15 \mathrm{~b}$, were overlaid and confirm that consistent pulse characteristics were being obtained, i.e. that time intervals between pulses are the same and both $T_{r}$ and $T_{F W H M}$ of detected pulse increases with propagation distance.

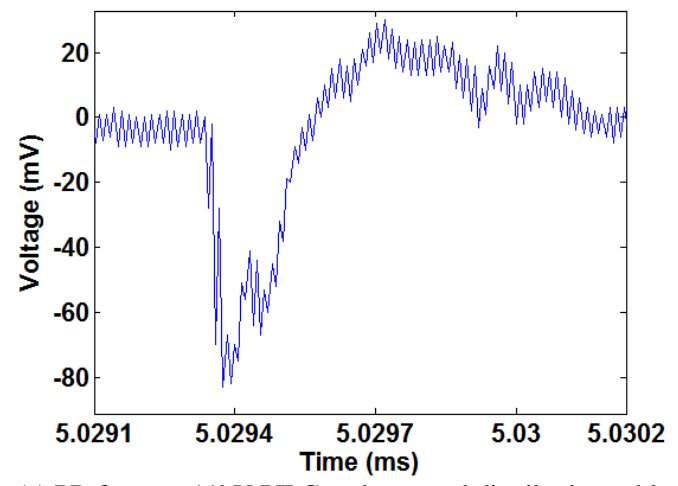

(a) PD from an 11kV PILC underground distribution cable

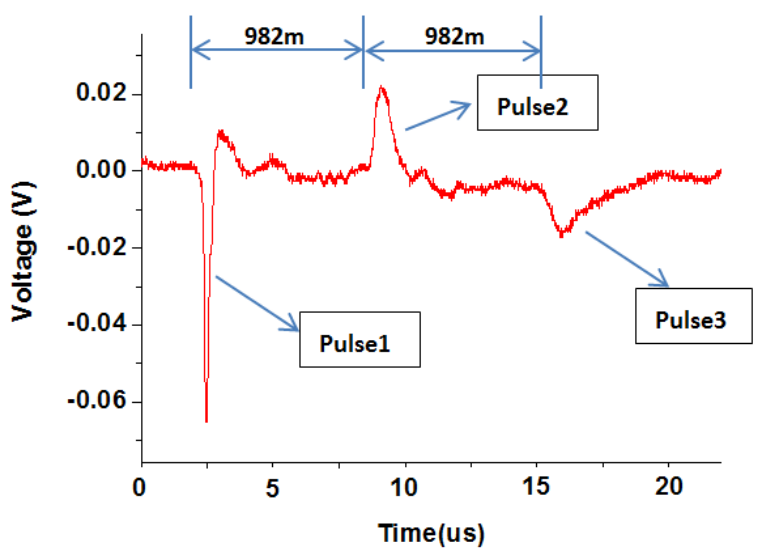

(b) PD from an $11 \mathrm{kV}$ EPR cable in a power station

Figure 15. On-site measurement data of PD pulses (sample rate is $100 \mathrm{MS} / \mathrm{s}$ )

\subsection{MEASURED ON-SITE INTERFERENCE SIGNALS}

Different types of noise, both pulsative and non-pulsative, can be present in measurements. The authors have extensively studied and developed techniques for denoising and identifying PD in cables, such as matched filters, digital filters, wavelet transform (WT) and second generation wavelet transform (SGWT) [22, 23]. However, these denoising techniques are not completely suitable for application to signals prior to locating faults, as they can modify the pulse characteristics. The method given below can be applied for location of PD sources using the original signal, once denoising has identified the pulses to be analyzed.

The presence of interference signals during on-site PD measurement is provided in Figure 16. Figure 16a shows a typical signal from an $11 \mathrm{kV}$ PILC underground distribution cable, Figure $16 \mathrm{~b}$ is a typical signal from an $11 \mathrm{kV}$ EPR cable in a power station in the UK. A comparison of the frequency spectrum of detected interference signals, i.e. which has been attenuated by both the cable and PD monitoring system, and the transfer functions of the HFCT and filter are shown in Figure 17. Also shown in Figure 17 are signals expected from PD pulses after propagation in a cable and through the detection system. The blue line is the frequency spectrum of a pulse after propagating along a $50 \mathrm{~m}$ cable while the red line is that of the pulse after propagation through a $50 \mathrm{~m}$ cable, a HFCT and a filter. Their high frequency spectra are almost the same, but the low frequency spectrum is obviously different which results from the frequency response of the HFCT and the filter. It can be seen that, in addition to the differences in time domain, the interference signals and detected PD pulses possess different frequency spectrum. This difference should prove useful in aiding to distinguish between PD pulses and noise.
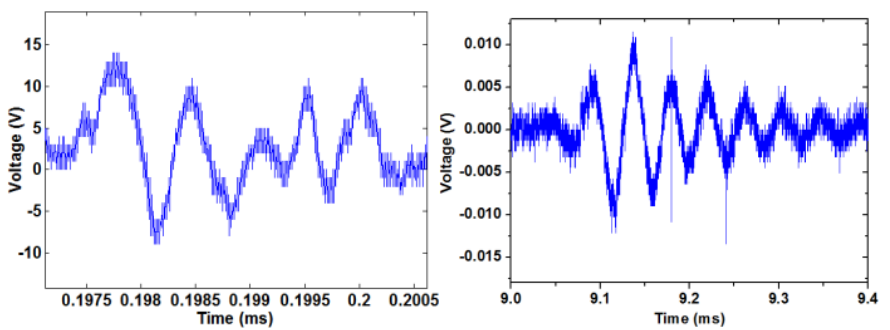

(a) Signal from an $11 \mathrm{kV}$ PILC cable (b) Signal from an $11 \mathrm{kV}$ EPR cable Figure 16. On-site measured interference signals 


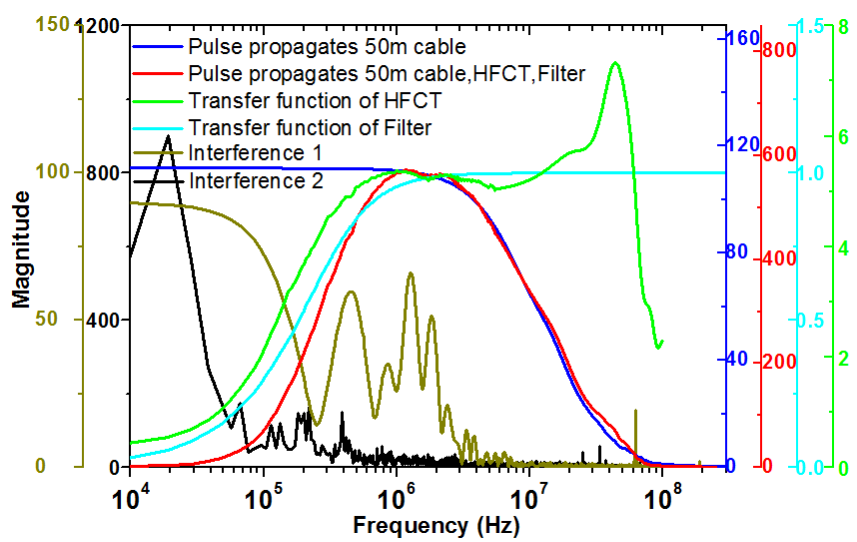

Figure 17. Comparison of the frequency spectra of detected interference signals, the transfer functions of HFCT and filter and signals expected from $\mathrm{PD}$ pulses after propagation in cable and through the detection system

\section{CONCLUSION AND FUTURE WORK}

PD monitoring systems capable of providing on-line data are normally comprised of HFCT and a high-pass filter. The detection system, in addition to the frequency dependent attenuation by the cable being monitored, results in the detected PD pulse characteristics being distorted. Simulations of signal modification, based on the frequency analyses using transfer functions of the cable, the HFCT and the filter, have been verified by experiments in laboratory and on-site PD measurements.

Based on the investigation, PD pulse propagation in a cable and PD monitoring system has the following characteristics:

The rise time and the pulse width of a PD pulse increase monotonically as it propagates along a cable.

An inverse peak is often associated with the PD pulse when detected by the PD monitoring system. The magnitude of the inverse pulse is affected by the detected pulse frequency bandwidth, which will be reduced due to the fact that the lower end of the frequency spectrum is attenuated heavily by the HFCT and the filter and the higher end frequencies by the cable, the phase shift introduced in the transfer function will also impact on the pulse shape.

Due to the impact of the cable and detection system, the further a pulse propagates, the greater is the ratio of the first inverse peak to the first peak of detected pulse (FIP-FP). This can be developed for PD localization.

The pulse bandwidth becomes limited to $20 \mathrm{MHz}$ after a pulse travels a distance of approximately $50 \mathrm{~m}$ in an $11 \mathrm{kV}$ EPR cable. This indicates that the detected pulse shape, when using a sensor/detection system with an upper cut-off frequency of $20 \mathrm{MHz}$, would be modified by the sensor/detection system if the source of PD is shorter than 50m away from the sensor.

Although many techniques for denoising in cable PD detection, such as matched filters, digital filters, wavelet transform (WT) and second generation wavelet transform (SGWT) have been developed, these denoising techniques require prior knowledge on the shape of the PD pulses and their differences with the pulse-shaped noises. The outcome of the paper will help to further improve denoising algorithms in practical applications.
Future work, based on the outcomes of this work, are to develop a novel on line PD localization technique based on the characteristics of pulse propagation in cable and PD monitoring system.

\section{REFERENCES}

[1] Fukunaga, K., Tan, M., Takehana, H., "New partial discharge detection method for live UHV/EHV cable joints", Electrical Insulation, IEEE Transactions on, Volume: 27, Issue: 3, 669-674, 1992.

[2] C. Zhou et al, 2012, "Statistical approaches for analysis of condition monitoring and failure data in power cables", CIGRE D1-314, Paris.

[3] Mansor, M., "Classification of PD patterns in XLPE cables under various soil conditions using statistical technique", Conference Record of the 2002 IEEE International Symposium on Electrical Insulation, USA.

[4] J. Densley, "Ageing mechanisms and diagnostics for power cables-an overview”, IEEE Electr. Insul. Mag., Vol. 17, No. 1, pp. 14-22, 2001.

[5] Van der Wielen, P., Steennis, E.F., Wouters, P.A.A.F., "Fundamental aspects of excitation and propagation of on-line partial discharge signals in three-phase medium voltage cable systems" Dielectrics and Electrical Insulation, IEEE Transactions on, Volume: 10, Issue: 4, 678 - 688, 2003.

[6] S. Boggs and J. Densley, "Fundamentals of Partial Discharge in the Context of Field Cable Testing", Electrical Insulation, IEEE Transactions on, Volume: 16, Issue: 5, 13-18, 2000.

[7] Van der Wielen, P., Steennis, E.F., "A centralized condition monitoring system for MV power cables based on on-line partial discharge detection and location", 2008 international conference on condition monitoring and diagnosis, China, 2008.

[8] Zhou, C., Song, X., Michel, M., Hepburn, D.M., "On-line partial discharge monitoring in medium voltage underground cables", Science, Measurement \& Technology, IET, Volume: 3 , Issue: 5, $354-363$, 2009.

[9] Weissenberg, W., Farid, F., Plath, R., Rethmeier, K., "On-site PD detection at cross-bonding links of HV cables", CIGRE, Paris, 2004.

[10] J. Veen and P.C.J.M. van der Wielen, "The application of matches filters to PD detection and localization", IEEE Electrical Insulation, Volume: 19, Issue: 5, 20-26, 2003.

[11] Van der Wielen, P.C.J.M., Steennis, E.F., "On-line PD monitoring system for MV cable connections with weak spot location", Power and Energy Society General Meeting, USA, 2008.

[12] Oussalah, N., Zebboudj, Y., Boggs, S.A., "Partial Discharge Pulse Propagation in Shielded Power Cable and Implications for Detection Sensitivity", Electrical Insulation Magazine, IEEE, Volume: 23 , Issue: 6, 5-10, 2007.

[13] Jim Jun Guo, Boggs, S.A., "High Frequency Signal Propagation in Solid Dielectric Tape Shielded Power Cables", Power Delivery, IEEE Transactions on, Volume: 26, Issue: 3, 1793 - 1802, 2011.

[14] Okubo, H., Hayakawa, N., "A novel technique for partial discharge and breakdown investigation based on current pulse waveform analysis", Dielectrics and Electrical Insulation, IEEE Transactions on, Volume: 12 , Issue: 4, 736 - 744, 2005.

[15] Papazyan, R., Pettersson, P., Edin, H., Eriksson, R., Gafvert, U., "Extraction of high frequency power cable characteristics from Sparameter measurements", Dielectrics and Electrical Insulation, IEEE Transactions on, Volume: 11, Issue: 3, 461 - 470, 2004.

[16] Cavallini, A., Montanari, G.C., Puletti, F., "A novel method to locate PD in polymeric cable systems based on amplitude-frequency (AF) map", Dielectrics and Electrical Insulation, IEEE Transactions on, Volume: 14 , Issue: 3, 726-734, 2007.

[17] Papazyan, R., Eriksson, R., "Calibration for time domain propagation constant measurements on power cables", Instrumentation and Measurement, IEEE Transactions on, Volume: 52 , Issue: 2, 415 - 418, 2003.

[18] Oussalah, N., Zebboudj, Y., Boggs, S.A., ”Analytic solution for pulse propagation in shielded power cable for sysmmetric and asymmetric PD pulses", Dielectrics and Electrical Insulation, IEEE Transactions on, Volume: 14, Issue: 5, $1264-1270,2007$.

[19] S. A. Boggs and G. C. Stone, "Fundamental limitations in the measurement of corona and partial discharge", IEEE Electrical Insulation, Volume: EI-17, Issue: 2, 143-150, 1982. 
[20] Yoshida, H., Tian, Z., Hikita, M., Miyata, H., "Study on the time evolution of partial discharge characteristics and interfacial phenomena of simulated XLPE cable joint", Proceedings of 1998 international symposium on electrical insulating materials, Page(s): 501 - 504, Japan, 1998.

[21] Gaxiola, E.H.R., Wetzer, J.M., "Partial discharge modelling and measurements on micrometer voids in solid dielectrics", Dielectric Materials, Measurements and Applications, Seventh International Conference on, Page(s): 322 - 325, Sep. 1996.

[22] Xiaodi Song, Chengke Zhou, Hepburn, D.M., Guobin Zhang, Michel, M, "Second generation wavelet transform for data denoising in PD measurement", IEEE transactions on Dielectrics and Electrical Insulation, Volume: 14, Issue: 6, 1531-1537, 2007.

[23] C. Zhou, X. Zhou, B.G. Stewart, A. Nesbitt, D.M. Hepburn, D. Guo, M. Michel, "Comparisons of Digital Filter, Matched Filter and Wavelet Transform in PD Detection”, CIGRE 2006.

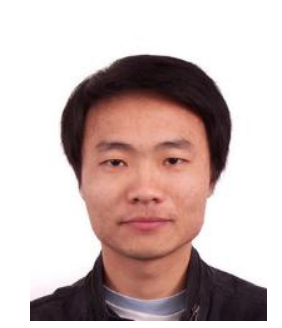

Bojie Sheng (M'13) was born in China. He received his B.Sc. degree from School of Electrical Engineering, Wuhan University, China in 2008. He is now a PhD candidate in School of Engineering and the Built Environment, Glasgow Caledonian University, UK and his research is mainly focused on the diagnostic methodology for power cable insulation as well as lightning protection for microelectronic equipment.

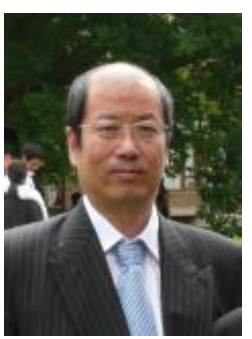

Chengke Zhou (M'06, SM'13) received the B.Sc. and M.Sc. degrees in electrical engineering from HuaZhong University of Science and Technology, China in 1983 and 1986, respectively, and the Ph.D. degree at the University of Manchester U.K., in 1994. Since then, he worked in Glasgow Caledonian University (GCU), as a Lecturer and Senior Lecturer, and in Heriot-Watt University as a Reader. In 2007 he returned to GCU as a Professor. He has published more than 100 papers in the area of PD based condition monitoring of MV/HV plant and power system analysis. He is Senior member of IEEE and Fellow of IET.

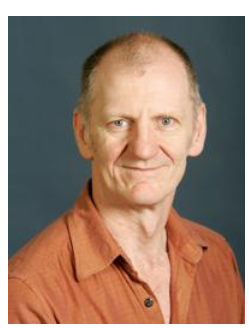

Donald M. Hepburn (M'08) received his B.A. (Hons) from the Open University in 1987and Ph.D. degree from Glasgow Caledonian University (GCU) in 1994. $\mathrm{He}$ is a Senior Lecturer at GCU, a member of the IEEE, Institute of Physics, the IET and C.Eng. He has 20 years of industrial research experience and has been involved in research into HV insulation systems at GCU for over 20 years. His research interests cover monitoring physical and chemical change in HV/MV insulation materials and application of advanced digital signal processing to information from electrical, acoustic and RF monitoring techniques. 